\title{
Interleukin-10 endogenously expressed in microglia prevents lipopolysaccharide-induced neurodegeneration in the rat cerebral cortex in vivo
}

\author{
Keun Woo Park ${ }^{1,2 *}$, Hwan Goo Lee ${ }^{1,2 *}$, \\ Byung Kwan Jin ${ }^{1,2,3}$ and Yong Beom Lee, $e^{1,2,3,4}$ \\ ${ }^{1}$ Neuroscience Graduate Program \\ ${ }^{2}$ Division of Cell Transformation and Restoration \\ ${ }^{3}$ Brain Disease Research Center \\ Ajou University School of Medicine \\ Suwon 443-721, Korea \\ ${ }^{4}$ Corresponding author: Tel, 82-31-219-4558; \\ Fax, 82-31-216-6381; E-mail, yblee@ ajou.ac.kr \\ *These authors contributed equally to this work.
}

Accepted 19 November 2007

Abbreviations: DAB, diaminobenzidine; GFAP, glial fibrillary acidic protein; IL-10NA, IL-10 neutralizing antibody; NO, nitric oxide; PB, phosphate buffer; ROS, reactive oxygen species

\begin{abstract}
A degree of brain inflammation is required for repair of damaged tissue, but excessive inflammation causes neuronal cell death. Here, we observe that IL-10 is expressed in LPS-injected rat cerebral cortex, contributing to neuronal survival. Cells immunopositive for IL-10 were detected as early as $8 \mathrm{~h}$ post-injection and persisted for up to $3 \mathrm{~d}$, in parallel with the expression of IL-1 $\beta$, TNF- $\alpha$, and iNOS. Double immunofluorescence staining showed that IL-10 expression was localized mainly in activated microglia. Next, we examined the neuroprotective effects of IL-10 using IL-10 neutralizing antibody (IL-10NA). Blockade of IL-10 action caused a significant loss of neurons both $3 \mathrm{~d}$ and $7 \mathrm{~d}$ after LPS injection. Further, the induction of mRNA species encoding IL-1 $\beta$, TNF- $\alpha$, and iNOS, reactive oxygen species (ROS) production, and NADPH oxidase activation, increased after co-injection of LPS and IL-10NA, compared to the levels seen after injection of LPS alone. Taken together, these results clearly suggest that LPS-induced endogenous expression of IL-10 in microglia contributes to neuronal survival by inhibiting brain inflammation.
\end{abstract}

Keywords: inflammation; interleukin-10; lipopolysaccharide; microglia; NADPH oxidase; neuron; reactive oxygen species

\section{Introduction}

It is well known that brain inflammation is a doubleedged sword. When brain inflammation is controlled and well-balanced, the inflammation significantly contributes to repair of damaged tissue after brain injury (Nguyen et al., 2002; Martino, 2004; Glezer et al., 2007). However, excessive inflammation damages surrounding healthy tissue, and such inflammation is now considered to be actively involved in both acute and chronic neurological disorders (Zipp and Aktas, 2006; Perry et al., 2007). Inflammatory responses in the brain are associated mainly with microglia, which are major immune effector cells in the CNS (Kreutzberg, 1996; Block et al., 2007). Activated microglia release pro-inflammatory and cytotoxic factors, including IL-1 $\beta$, IL-6, TNF- $\alpha$, nitric oxide (NO), ROS, and arachidonic acid metabolites (Aloisi, 2001). These factors are necessary for the normal functions of microglia in the immune responses of the CNS, and also for CNS repair. It follows that the extent of microglial activation should be strictly controlled (Hanisch and Kettenmann, 2007). Thus, understanding how the extent and duration of brain inflammation are controlled in vivo is important from the standpoint of pathophysiology.

IL-10, an immunosuppressive cytokine, is known to be produced by both human and murine microglia when these cells are stimulated with LPS and/or IFN- $\gamma$ (Mizuno et al., 1994; Williams et al., 1996; Lee et al., 2002; Seo et al., 2004), and IL-10 production in turn suppresses the production of pro-inflammatory mediators such as IL-1 $\beta$, TNF$\alpha$, and iNOS, in cell cultures (Aloisi et al., 1999a; Ledeboer et al., 2000). IL-10 is therefore considered to be an important anti-inflammatory modulator of glial activation, functioning to maintain a balance between pro- and anti-inflammatory cytokine levels in the CNS (Sawada et al., 1999). Moreover, two recent studies have shown that treatment with IL-10 inhibited LPS-induced degeneration of dopaminergic neurons either in culture, or in the substantia nigra, by preventing microglial activation (Qian et al., 2006; Arimoto et al., 2007). Collectively, these observations suggest that IL-10 produced by microglia counteracts brain inflammation, and that such production leads to neuronal survival. Little is known, however, regarding when 
or how microglial activation is suppressed upon inflammation of the brain parenchyma.

In the present study, we therefore examined whether LPS injection into rat cerebral cortex induces endogenous expression of IL-10 in microglia in vivo. We also sought to determine whether IL-10 contributes to the downregulation of inflammation, and whether IL-10 might thus increase neuronal survival.

\section{Materials and Methods}

\section{Animal treatments}

Female Sprague-Dawley rats (230-280 g) were anesthetized with chloral hydrate $(400 \mathrm{mg} / \mathrm{kg}$ i.p.), positioned in a stereotaxic apparatus, and LPS (Sigma, St. Louis, MO) was delivered unilaterally into the right cerebral cortex (anteroposterior 1.4 $\mathrm{mm}$, mediolateral $2.0 \mathrm{~mm}$, dorsoventral $2.0 \mathrm{~mm}$ from bregma). $5 \mu \mathrm{g}$ of LPS was injected at a rate of $0.2 \mu \mathrm{l} / \mathrm{min}$ with a 30 -gauge Hamilton syringe attached to an automated microinjector (Buwon, Seoul, Korea). For neutralization of IL-10, some animals received co-injection of LPS and antimurine IL-10 neutralizing antibody or nonspecific murine IgG as a control ( $1 \mu \mathrm{g}$; R\&D Systems, Minneapolis, $\mathrm{MN}$ ).

\section{Immunohistochemistry}

Brain sections were incubated with the appropriate primary antibodies against mouse OX-42 (1:400; Serotec, Oxford, UK) for microglia, against mouse glial fibrillary acidic protein (GFAP, 1:500; Sigma) for astrocytes, and against NeuN (1:500; Chemicon International, Temecula, CA) for neurons. The following day, sections were incubated with appropriate biotinylated secondary antibody followed by avidin-biotin complex (Elite Kit from Vector Laboratories, Burlingame, CA). The bound antiserum was visualized by incubating with $0.05 \%$ diaminobenzidine- $\mathrm{HCl}(\mathrm{DAB})$ and $0.003 \%$ hydrogen peroxide in $0.1 \mathrm{M}$ phosphate buffer (PB).

\section{Double-immunofluorescence staining}

For immunofluorescence staining, the brain sections were incubated with a combination of a mouse OX-42 (1:400) and goat anti-IL-10 (1:150); mouse anti-GFAP (1:400; Sigma) and goat antiIL-10; mouse anti-NeuN (1:400) and goat anti-IL10; mouse OX-42 and goat anti-IL-1 $\beta$ (1:200; R\&D Systems); mouse OX-42 and rabbit anti-iNOS (1:200; Chemicon); mouse OX-42 and goat antiTNF- $\alpha$ (1:50; R\&D Systems). After washing, the sections were subsequently incubated with FITClabeled anti-mouse IgG (1:200; Kirkegaard \& Perry Laboratories, Gaithersburg, MD) and Texas redlabeled anti-rabbit $\lg G(1: 200 ;$ Vector $)$ or Texas Red-labeled anti-goat IgG (1:200; Vector). Tissues were mounted with Vectashield mounting medium (Vector) and viewed using an Olympus IX71 confocal laser scanning microscope (Olympus; Tokyo, Japan).

\section{RT-PCR}

Brains from the ipsilateral cortex after injection were used for RNA isolation using Trizol (Life Technology, MD). The reverse transcribed cDNA (2 $\mu \mathrm{g}$ of RNA) was subjected to PCR amplification with following primer sets. For rat IL-10, 5'-TGCCTTCAGTCAAGTGAAGAC-3' (sense) and 5'-AAACTCATTCATGGCCTTGTA-3' (antisense) and for rat IL-1 $\beta$, 5'-CTCCATGAGCTTTGTACAAGG-3' (sense) and 5'-TGCTGATGTACCAGTTGGGG-3' (antisense) and for rat TNF- $\alpha$, 5'-GTAGCCCACGTCGTAGCAAA-3' (sense) and 5'-CCCTTCTCCAGCTGGGAGAC-3' (antisense) and for rat iNOS, 5'-CACCTACTTCCTGGACATCACTAC- 3' (sense) and 5'-GTACTCTGAGGGCTGACACAAG-3' (antisense). The PCR cycles consisted of denaturation at $94^{\circ} \mathrm{C}$ for $30 \mathrm{~s}$, annealing at $57^{\circ} \mathrm{C}$ for $30 \mathrm{~s}$ (TNF- $\alpha, \mathrm{IL}-10, \mathrm{IL}-1 \beta$ ) or $60^{\circ} \mathrm{C}$ for $30 \mathrm{~s}$ (iNOS), and extension at $72^{\circ} \mathrm{C}$ for $90 \mathrm{~s}$ for 25 cycles. GAPDH was also amplified as an internal PCR control using the following primers, 5'-TCCATGACAACTTTGGCATCGTGG-3' (sense) and 5'-GTTGCTGTTGAAGTCACAGGAGAC-3' (antisense). PCR products were separated by electrophoresis, stained with ethidium bromide, and then detected using UV light.

\section{In situ detection of $\mathrm{O}_{2}^{-}$and $\mathrm{O}_{2}^{-}$-derived oxidants}

Hydroethidine histochemistry was performed for in situ visualization of $\mathrm{O}_{2}{ }^{-}$and $\mathrm{O}_{2}{ }^{-}$-derived oxidants (Wu et al., 2003). After injection, hydroethidine (1 $\mathrm{mg} / \mathrm{ml}$; Molecular Probes, Eugene, OR) was administered intraperitoneally. The brain sections with oxidized hydroethidine product, ethidium, were examined by confocal microscopy (Olympus).

\section{Western blot analysis}

For subcellular fractionation, protein extracts of both the cytosolic and membrane factions were prepared. Protein $(30 \mu \mathrm{g})$ were separated by SDSPAGE and transferred to polyvinylidene difluoride membranes (Millipore, Bedford, MA). The membranes were incubated with following primary anti- 
bodies: mouse anti-Rac1 (1:1,000; BD Biosciences, San Diego, CA), mouse anti-p67phox (1:1,000; BD Biosciences), mouse anti-p47phox (1:1,000; BD Biosciences), and mouse anti- $\beta$-actin $(1: 5,000$; Sigma), and rabbit anti-calnexin (1:1,000; Santa Cruz Biotechnology, Santa Cruz, CA). After washing, the membranes were incubated with secondary antibodies (1:2,000; Amersham Pharmacia, Buckinghamshire, UK). Finally, the blots were developed with enhanced chemiluminescence detection reagents (Amersham Pharmacia).

\section{Statistical analysis}

All values are represented as mean \pm SEM. Statistical significance ( $P<0.05$ for all analyses) was assessed by ANOVA using Instat 3.05 (GraphPad Software, San Diego, CA), followed by Student-Newman-Keuls analyses.

\section{Results}

\section{LPS induces microglial activation and expression of pro-inflammatory mediators in rat cerebral cortex}

After unilateral microinjection of LPS or PBS (as a control) into the cerebral cortex of rats, brain sections were processed for immunostaining using OX-42, which detects CR3 complement receptors. In PBS-injected cortex (24 h post-injection), most OX-42-immunopositive (OX-42-ip) microglia exhibited a resting morphology of small cell bodies and long ramified processes. In contrast, $24 \mathrm{~h}$ after intracortical injection of LPS, the morphology of OX-42-ip microglia changed to the activated form, consisting of enlarged cell bodies with short processes, and this morphology was maintained for up to $3 \mathrm{~d}$ after LPS injection (Figure 1A). Next, we examined the expression of pro-inflammatory mediators such as IL-1 $\beta$, TNF- $\alpha$, and iNOS, in LPS-injected rat cerebral cortex. The mRNAs encoding these factors were induced as early as $4 \mathrm{~h}$ after LPS injection, and peaked at $12 \mathrm{~h}$, returning to normal levels $7 \mathrm{~d}$ post-injection (Figure $1 \mathrm{~B}$ and C), indicating that inflammation was subject to endogenous control.

\section{IL-10 is expressed in LPS-injected cerebral cortex in parallel with the expression of pro-inflammatory mediators}

Next, we examined whether LPS might induce IL10 expression in vivo by unilateral injection of LPS into rat cerebral cortex. Expression of IL-10 mRNA was detected as early as $4 \mathrm{~h}$ after LPS injection and this expression was maintained for up to $3 \mathrm{~d}$ post-injection, returning to normal levels $7 \mathrm{~d}$ postinjection (Figure 2A). IL-10-specific immunohistochemistry revealed that IL-10-immunopositive cells were detected as early as $8 \mathrm{~h}$ after LPS injection,

$\mathbf{A}$

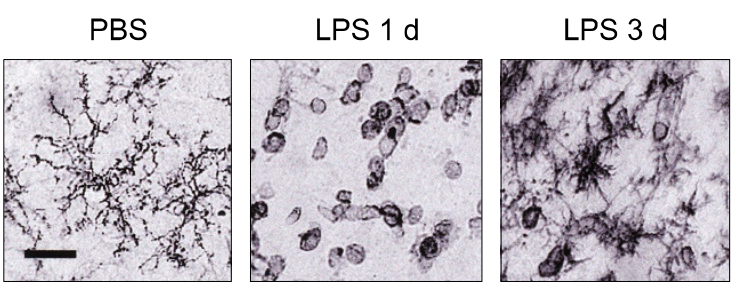

B
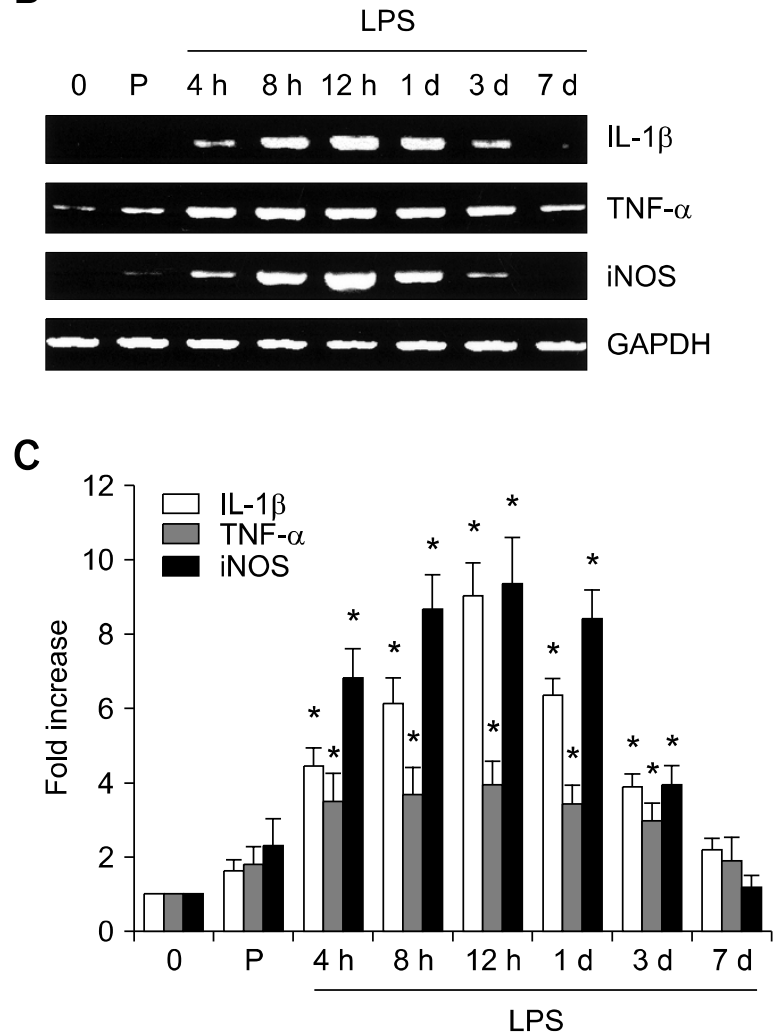

Figure 1. Microglial activation and expression of proinflammatory cytokine after unilateral injection of LPS. (A) LPS $(5 \mu \mathrm{g} / 3 \mu \mathrm{l})$ or PBS was injected into the cerebral cortex. Animals were killed at $1 \mathrm{~d}$ or $3 \mathrm{~d}$ after injection, brains were removed, and coronal sections $(40 \mu \mathrm{m})$ were cut using sliding microtome. Every sixth serial sections were selected and processed for OX-42 immunostaining for microglia. Note morphological changes in microglia from resting (small cell bodies and thin, long, or ramified processes) to activated state (larger cell bodies with no processes). Scale bar, $150 \mu \mathrm{m}$. (B) RT-PCR analysis of LPS-induced mRNA expression of proinflammatory cytokines, TNF- $\alpha, \mathrm{IL}-1 \beta$, and iNOS in the cerebral cortex. Animals were decapitated after injection of LPS, and total RNA was isolated in the injected area of cortex at indicated time points. (C) Error bars represent the mean \pm SEM from three samples per each time point. ${ }^{*} P<0.001$ compared with control according to ANOVA and Student- Newman-Keuls analyses. 
A
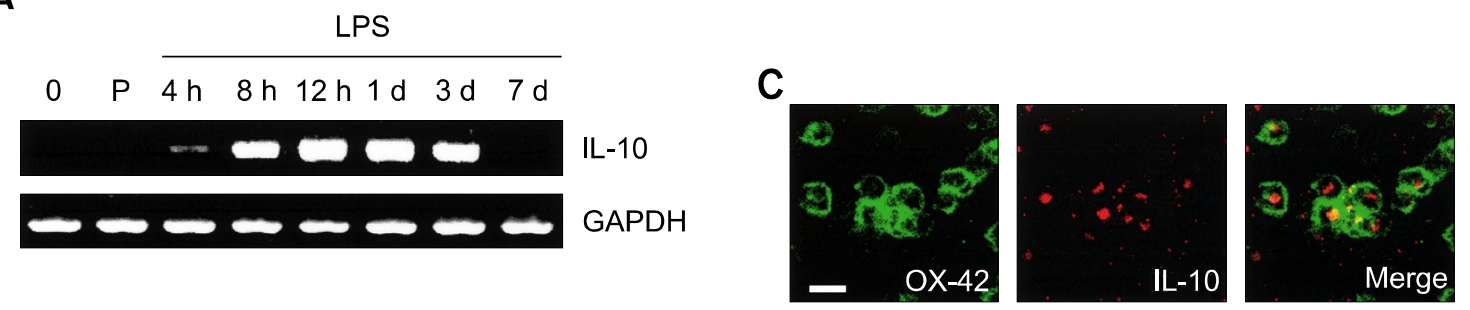

B
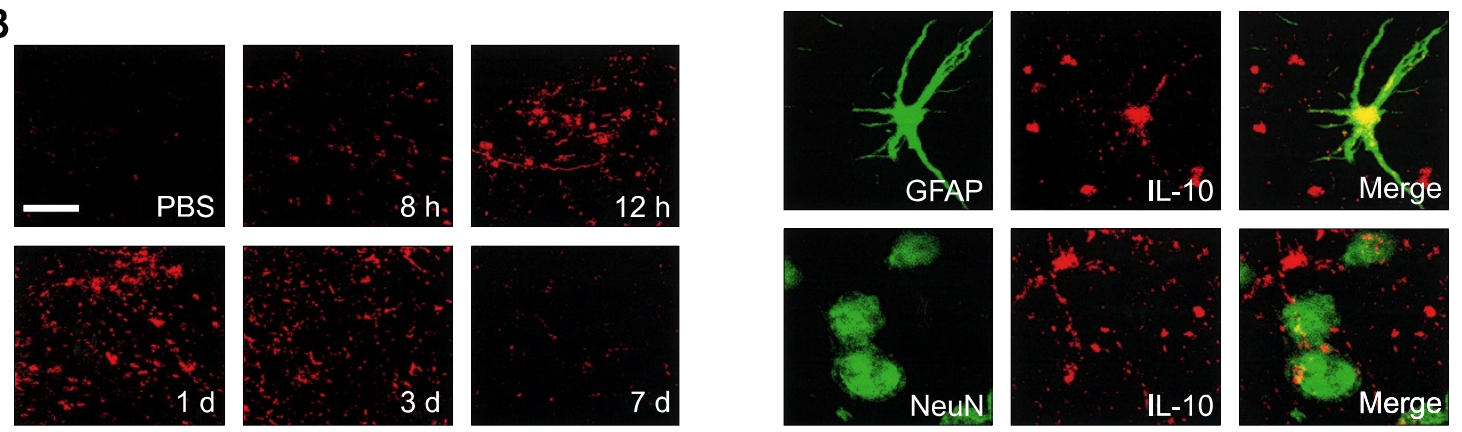

Figure 2. LPS-induced IL-10 expression and co-localization within activated microglia and astroyctes. (A) RT-PCR analysis of LPS-induced mRNA expression of IL-10 in the cerebral cortex. Animals were decapitated after injection of LPS, and total RNA was isolated in the injected area of cortex at indicated time points. (B) PBS or LPS $(5 \mu \mathrm{g} / 3 \mu \mathrm{l})$ was unilaterally injected into the rat cerebral cortex. Animals were sacrificed at various time points, brains were removed, and coronal sections $(40 \mu \mathrm{m})$ were cut with a microtome. Every sixth serial section was selected and processed for immunostaining with IL-10 antibody at $8 \mathrm{~h}, 12 \mathrm{~h}, 1 \mathrm{~d}, 3 \mathrm{~d}$, and $7 \mathrm{~d}$ post-injection. (C) Double-immunofluorescence staining with antibodies to OX-42 for microglia and IL-10, glial fibrillary acidic protein (GFAP) for astrocytes and IL-10, or NeuN for neurons and IL-10 at $24 \mathrm{~h}$ after intracortical injection of LPS. Images from the double-labled tissue were merged. Scale bar, $10 \mu \mathrm{m}$.

were detectable for up to $3 \mathrm{~d}$ post-injection, but were no longer seen $7 \mathrm{~d}$ post injection (Figure 2B), indicating that IL-10 expression in LPS-injected cerebral cortex occurred in parallel with the expression of pro-inflammatory mediators. In PBSinjected control tissue, IL-10 protein expression was not detected.

To identify the specific cell types expressing IL-10 in the cerebral cortex, double immunofluorescence staining for IL-10 and OX-42 (a microgliaspecific stain), for IL-10 and GFAP (an astrocytespecific stain), or for IL-10 and NeuN (a neuronspecific stain) was performed $24 \mathrm{~h}$ after LPS injection. Fluorescence images from each channel of the double-labeled sections were merged. The results showed that LPS-induced IL-10 expression was localized mainly in activated microglia, and in a few astrocytes, but not in neurons (Figure 2C).

\section{Blockade of IL-10 action exacerbates neuronal cell death in the cerebral cortex}

To confirm that IL-10 endogenously produced in microglia prevented LPS-induced neurodegeneration in the rat cerebral cortex in vivo, we measured neuronal cell death $1 \mathrm{~d}, 3 \mathrm{~d}$, and $7 \mathrm{~d}$ after co-injection of LPS and IL-10NA, using animals injected with LPS only as controls. We observed significant losses of NeuN-ip neurons $3 \mathrm{~d}$ and $7 \mathrm{~d}$ after LPS and IL-10NA co-injection, compared to losses seen after injection of LPS only (Figure $3 A$ ). Injection of nonspecific murine $\operatorname{lgG}$ had little effect (data not shown). To delineate the site and extent of injury induced by injection of LPS alone or LPS and IL-10NA together into the cortex, a schematic drawing was made from cortical tissue immunostained with the NeuN antibody using a camera lucida microscope attachment. This showed that NeuN-ip cells disappeared in approximately $18 \%$ of the cortex in animals injected with both LPS and IL-10NA, whereas the corresponding figure in animals injected with LPS only was $2 \%$ compared to the PBS injected tissues (Figure 3B). These results clearly showed that IL-10 produced by microglia in LPS-injected cerebral cortex blocked neuronal cell death.

\section{Blockade of IL-10 action upregulates expression of pro-inflammatory mediators and ROS production in LPS-injected cerebral cortex}

To further explore the mechanisms responsible for the neuroprotective effects of IL-10, we examined whether blockade of IL-10 action might result in the expression of higher levels of pro-inflammatory and cytotoxic factors than seen in animals injected with LPS only. As expected, prolonged induction of IL$1 \beta$, TNF- $\alpha$, and iNOS mRNA expression was ob- 
A

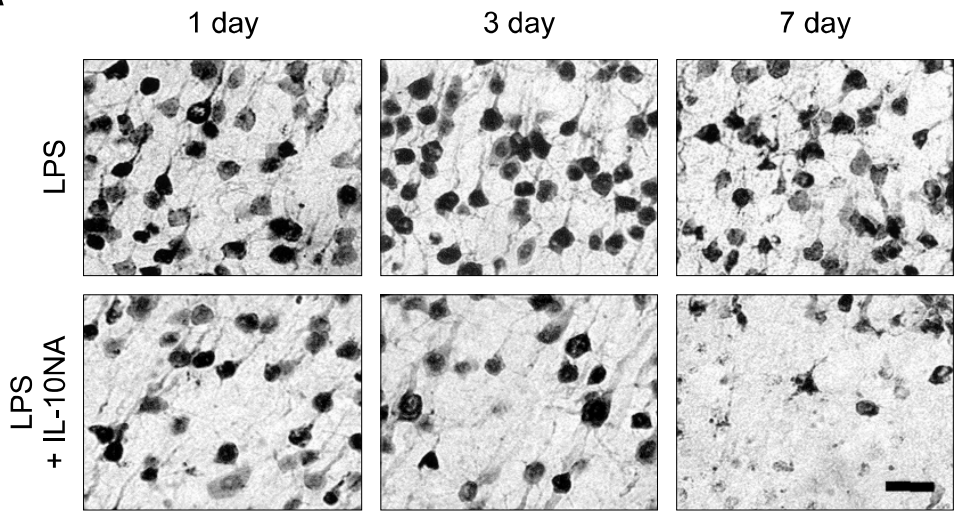

B

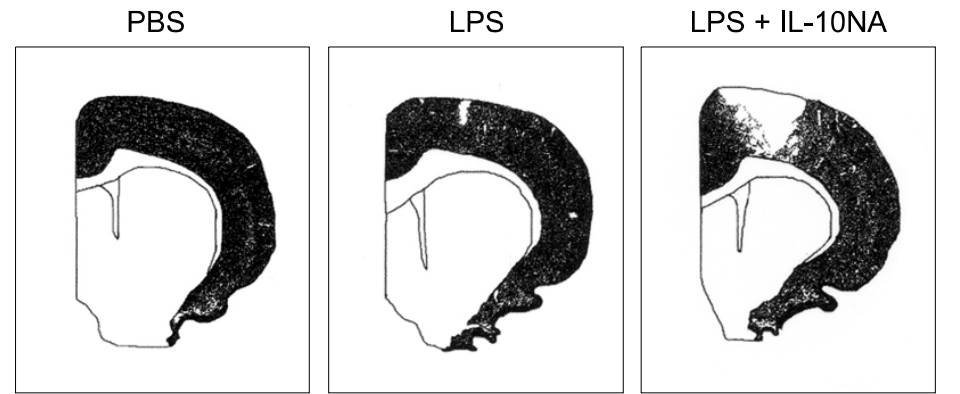

Figure 3. Neutralization of IL-10 exacerbated the death of cortical neurons after LPS injection in vivo. (A) $1 \mathrm{~d}, 3 \mathrm{~d}$, and $7 \mathrm{~d}$ after injection of LPS, or LPS plus IL-10NA, brain sections were processed for NeuN. Scale bar, $250 \mu \mathrm{m}$ (B) Schematic drawing of a representative coronal section from rat brain showing distribution of NeuN-ip neurons traced using a camera lucida microscope attachment $7 \mathrm{~d}$ after PBS, LPS, or LPS plus IL-10 NA injection.

served for up to $3 \mathrm{~d}$ after co-injection of LPS and IL-10NA, compared to the levels seen after injection of LPS only (Figure 4A and B). In parallel with the prolonged induction of these pro-inflammatory factors, blockade of IL-10 action enhanced ROS production $3 \mathrm{~d}$ after LPS injection (Figure 4C). Further, we confirmed that the amounts of $p 47^{\text {phox }}$, p $67^{\text {phox }}$, and Rac 1 in the membrane fraction increased at $24 \mathrm{~h}$, and were maintained to $72 \mathrm{~h}$, after co-injection of LPS and IL-10NA, compared to the protein levels seen after injection of LPS only (Figure 4D). These results suggested that the neuroprotective effects of IL-10 may be mediated by downregulation of pro-inflammatory mediators and inhibition of ROS production.

\section{Discussion}

In this study, we examined the endogenous expression of IL-10 in LPS-injected rat cerebral cortex, and found evidence of an association between IL-10 expression and downregulation of brain inflammation, leading to neuronal protection. IL-10 was expressed mainly in activated microglia, and blockade of IL-10 action resulted in upregulation of pro-inflammatory factors and ROS, exacerbating neuronal cell death.
It has been believed that inflammatory conditions created either in vivo or in vitro cause neurodegeneration through the release of inflammatory mediators and neurotoxic factors (Allan and Rothwell, 2001; Lucas et al., 2006). However, the control of neurotoxic functions under pathological conditions remains unexplained. In fact, brain parenchyma itself is able to control the extent of brain inflammation. In support of this, our results showed that upregulation of pro-inflammatory mediators in LPS-injected rat cerebral cortex was transient, and a return to normal levels was quickly attained. Although the mechanisms underlying the ability of the brain to control inflammation are poorly understood, it has been reported that microglia in culture expressed IL-10 following exposure to LPS (Williams et al., 1996; Seo et al., 2004 ), and that IL-10 could exert immunosuppressive properties by downregulating the expression of pro-inflammatory cytokines such as TNF- $\alpha$ and IL-12 by microglia (Aloisi et al., 1997, 1999b). Further, several studies have demonstrated neuroprotective effects of IL-10 against glutamate-mediated cerebellar granule cell death, hypoxic-ischemic neuronal cell death, and traumatic brain injury (Knoblach and Faden, 1998; Dietrich et al., 1999; Grilli et al., 2000; Bachis et al., 2001). These data strengthen the notion that the anti-inflammatory 
A

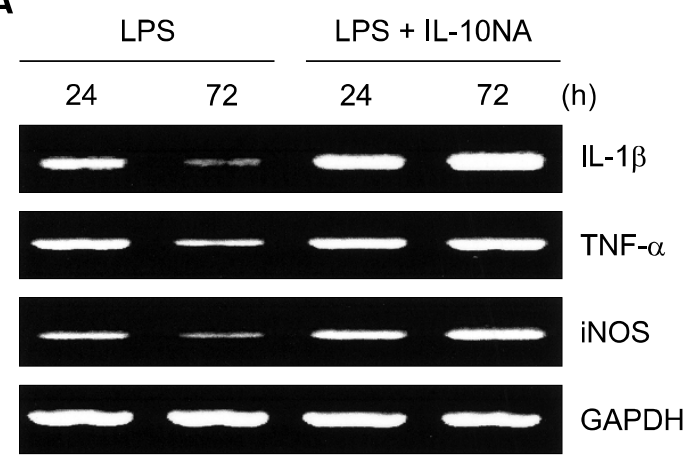

C

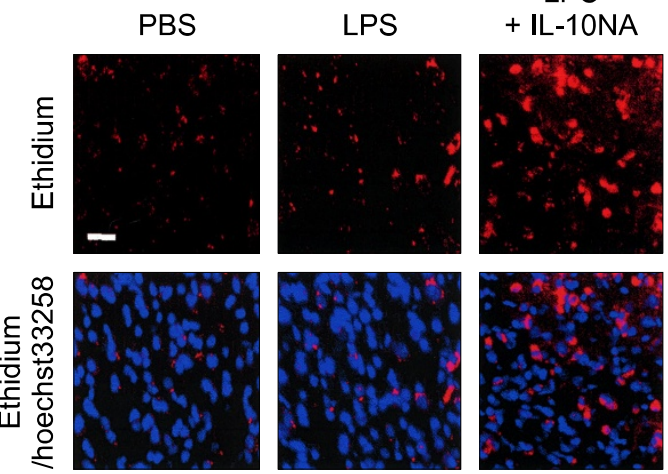

B

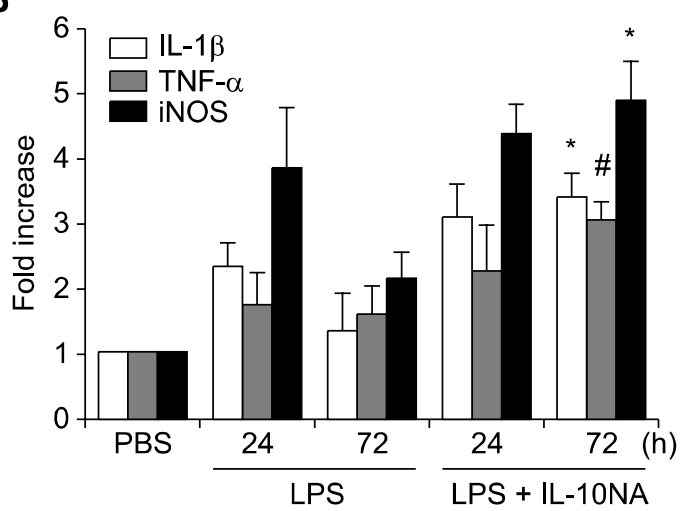

D

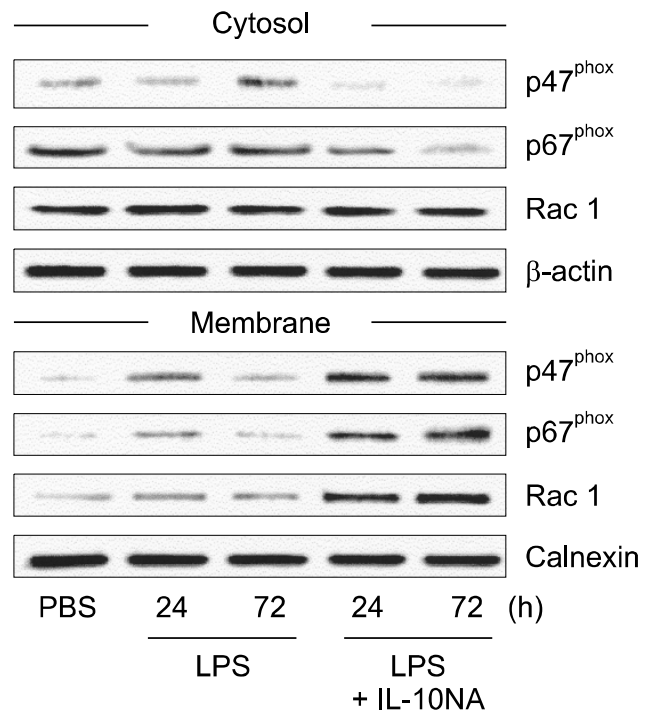

Figure 4. Neutralization of IL-10 led to the expression of proinflammatory cytokines and NADPH oxidase mediated production of ROS. (A) RT-PCR analysis of LPS-induced expression of proinflammatory cytokines, IL-1 $\beta$, iNOS, and TNF- $\alpha$ mRNA in the cerebral cortex in the absence or presence of IL-10NA in the rat cortex after LPS injection. (B) Error bars represent the mean \pm SEM from three samples per each time point. ${ }^{\#} P<0.01,{ }^{*} P<0.001$ compared with samples treated with LPS post $72 \mathrm{~h}$. (C) In situ visualization of $\mathrm{O}_{2}{ }^{-}$and $\mathrm{O}_{2}{ }^{-}$-derived oxidant production. Animals were injected with hydroethidine $3 \mathrm{~d}$ after intracortical injection of PBS or LPS $(5 \mu \mathrm{g} / 3 \mu \mathrm{l})$ in the absence or presence of IL-10NA $(1 \mu \mathrm{g} / \mathrm{ml})$. Confocal micrographs show ethidium fluorescence (red). Nuclei were counterstained with Hoechst33258 (blue). Scale bar, $20 \mu \mathrm{m}$. (D) NADPH oxidase is activated by LPS, as evidenced by the translocation of the $\mathrm{p} 67^{\text {phox }}, \mathrm{p} 47^{\text {phox }}$, and Rac 1 subunit from the cytosol to the plasma membrane; this translocation was sustained by IL-10NA. Tissue lysates from injected area were prepared $24 \mathrm{~h}$ or $72 \mathrm{~h}$ after injection of PBS or LPS $(5 \mu \mathrm{g} / 3 \mu \mathrm{l})$ in the absence or presence of IL-10 NA $(1 \mu \mathrm{g} / \mu \mathrm{l})$. Fractionated proteins were analyzed by SDS-PAGE and subjected to immunoblotting with anti-p67 $7^{\text {phox }}$, p47 $7^{\text {phox }}$, and Rac 1 antibody. The blots were reprobed with antibodies against the calnexin membrane protein as loading controls and to exhibit fractionation efficiency.

function of IL-10 can prevent inflammation- ediated neuronal degeneration under pathological conditions.

Importantly, in the present study, we showed that IL-10 was expressed in LPS-injected cerebral cortex, and that blockade of IL-10 action both exacerbated the expression of pro-inflammatory and cytotoxic factors, and increased neuronal cell death. A recent study demonstrated that osmotic pump infusion of recombinant IL-10 protected LPSinduced dopaminergic neuronal cell death in the substantia nigra, by inhibiting microglial activation (Arimoto et al., 2007). Further, our study found that
IL-10 was endogenously expressed in microglia, which in turn prevented LPS-induced neurodegeneration in the rat cerebral cortex in vivo, and that the time course of IL-10 expression paralleled those of the pro-inflammatory mediators IL-1 $\beta$, TNF- $\alpha$, and iNOS. Because microglial cells are major pro-inflammatory cytokine-producing cells, microglia may control the production of inflammatory mediators in an autocrine manner by producing IL-10, resulting in neuroprotection even from the early stage of acute brain inflammation.

Although the mechanisms responsible for neuronal cell death in brain inflammation remain 
largely unknown, increasing evidences have shown that oxidative stress plays an important role in inflammation-mediated neurodegeneration (Qin et al., 2004; Mander and Brown, 2005; Wu et al., 2006). In the present study, we showed that blockade of IL-10 action upregulated LPS-induced NADPH oxidase activation and ROS production, indicating that the neuroprotective effects of IL-10 are mediated by inhibition of NADPH oxidase activation in brain inflammation. Our results are comparable to those of a recent report indicating that IL-10 treatment reduced both LPS-induced production of pro-inflammatory mediators and neurotoxicity, in mesencephalic neuron-glia cultures, through inhibition of NADPH oxidase activity (Qian et al., 2006). Apart from NADPH oxidase, pro-inflammatory mediators may be involved in LPS-induced neurotoxicity. It has been shown that the levels of IL-1 $\beta$, TNF- $\alpha$, and iNOS are upregulated under neuropathological conditions (Allan and Rothwell, 2001; Lucas et al., 2006). Our present data show that blockade of IL-10 action caused LPS-mediated prolonged induction of IL$1 \beta$, TNF- $\alpha$, and iNOS, indicating that the neuroprotective role of IL-10 in LPS-induced neurotoxicity is mediated through downregulation of proinflammatory mediators in the rat cerebral cortex in vivo.

In conclusion, our study provides evidences that IL-10 endogenously produced in microglia prevents LPS-induced neurodegeneration in the rat cerebral cortex in vivo. The finding that blockade of IL-10 action upregulated both LPS-induced NADPH oxidase activation and the expression of pro-inflammatory mediators supports the novel concept that microglia can act in an autocrine manner to limit potentially deleterious effects of inflammation under neuropathological conditions.

\section{Acknowledgment}

This work was supported by the Korea Science and Engineering Foundation (KOSEF) through the Brain Disease Research Center at Ajou University.

\section{References}

Allan SM, Rothwell NJ. Cytokines and acute neurodegeneration. Nat Rev Neurosci 2001;2:734-44

Aloisi F, Penna G, Cerase J, Menendez Iglesias B, Adorini $\mathrm{L}$. IL-12 production by central nervous system microglia is inhibited by astrocytes. J Immunol 1997;159:1604-12

Aloisi F, De Simone R, Columba-Cabezas S, Levi G. Opposite effects of interferon- $\gamma$ and prostaglandin E2 on tumor necrosis factor and interleukin-10 production in microglia: a regulatory loop controlling microglia pro- and anti-inflammatory activities. J Neurosci Res 1999a;56:57180

Aloisi F, Penna G, Polazzi E, Minghetti L, Adorini L. CD40CD154 interaction and IFN- $\gamma$ are required for IL-12 but not prostaglandin E2 secretion by microglia during antigen presentation to Th1 cells. J Immunol 1999b;162:1384-91

Aloisi F. Immune function of microglia. Glia 2001;36:165-79 Arimoto T, Choi DY, Lu X, Liu M, Nguyen XV, Zheng N, Stewart CA, Kim HC, Bing G. Interleukin-10 protects against inflammation-mediated degeneration of dopaminergic neurons in substantia nigra. Neurobiol Aging 2007;28: 894-906

Bachis A, Colangelo AM, Vicini S, Doe PP, De Bernardi MA, Brooker G, Mocchetti I. Interleukin-10 prevents glutamatemediated cerebellar granule cell death by blocking caspase3-like activity. J Neurosci 2001;21:3104-12

Block ML, Zecca L, Hong JS. Microglia-mediated neurotoxicity: uncovering the molecular mechanisms. Nat Rev Neurosci 2007;8:57-69

Dietrich WD, Busto R, Bethea JR. Postischemic hypothermia and IL-10 treatment provide long-lasting neuroprotection of CA1 hippocampus following transient global ischemia in rats. Exp Neurol 1999:158:444-50

Glezer I, Simard AR, Rivest S. Neuroprotective role of the innate immune system by microglia. Neuroscience 2007; 147:867-83

Grilli M, Barbieri I, Basudev H, Brusa R, Casati C, Lozza G, Ongini E. Interleukin-10 modulates neuronal threshold of vulnerability to ischaemic damage. Eur J Neurosci 2000;12: 2265-72

Hanisch UK, Kettenmann H. Microglia: active sensor and versatile effector cells in the normal and pathologic brain. Nat Neurosci 2007;10:1387-94

Knoblach SM, Faden Al. Interleukin-10 improves outcome and alters proinflammatory cytokine expression after experimental traumatic brain injury. Exp Neurol 1998;153:143-51

Kreutzberg GW. Microglia: a sensor for pathological events in the CNS. Trends Neurosci 1996;19: 312-8

Ledeboer A, Breve JJ, Poole S, Tilders FJ, Van Dam AM. Interleukin-10, interleukin-4, and transforming growth factor$\beta$ differentially regulate lipopolysaccharide-induced production of pro-inflammatory cytokines and nitric oxide in cocultures of rat astroglial and microglial cells. Glia 2000;30: 134-42

Lee YB, Nagai A, Kim SU. Cytokines, chemokines, and cytokine receptors in human microglia. J Neurosci Res 2002;69:94-103

Lucas SM, Rothwell NJ, Gibson RM. The role of inflammation in CNS injury and disease. Br J Pharmacol 2006;147:232-40

Mander P, Brown GC. Activation of microglial NADPH oxidase is synergistic with glial iNOS expression in inducing neuronal death: a dual-key mechanism of inflammatory neurodegeneration. J Neuroinflammation 2005;2:20-34 
Martino G. How the brain repairs itself: new therapeutic strategies in inflammatory and degenerative CNS disorders. Lancet Neurol 2004;3:372-8

Mizuno T, Sawada M, Marunouchi T, Suzumura A. Production of interleukin-10 by mouse glial cells in culture. Biochem Biophys Res Commun 1994;205:1907-15

Nguyen MD, Julien JP, Rivest S. Innate immunity: the missing link in neuroprotection and neurodegeneration? Nat Rev Neurosci 2002;3:216-27

Perry VH, Cunningham C, Holmes C. Systemic infections and inflammation affect chronic neurodegeneration. Nat Rev Immunol 2007;7:161-7

Qian L, Block ML, Wei SJ, Lin CF, Reece J, Pang H, Wilson B, Hong JS, Flood PM. Interleukin-10 protects lipopolysaccharide-induced neurotoxicity in primary midbrain cultures by inhibiting the function of NADPH oxidase. J Pharmacol Exp Ther 2006;319:44-52

Qin L, Liu Y, Wang T, Wei SJ, Block ML, Wilson B, Liu B, Hong JS. NADPH oxidase mediates lipopolysaccharide-induced neurotoxicity and proinflammatory gene expression in activated microglia. J Biol Chem 2004;279:1415-21

Sawada M, Suzumura A, Hosoya H, Marunouchi T, Nagatsu
T. Interleukin-10 inhibits both production of cytokines and expression of cytokine receptors in microglia. J Neurochem 1999;72:1466-71

Seo DR, Kim KY, Lee YB. Interleukin-10 expression in lipopolysaccharide-activated microglia is mediated by extracellular ATP in an autocrine fashion. Neuroreport 2004; 15:1157-61

Williams K, Dooley N, Ulvestad E, Becher B, Antel JP. IL-10 production by adult human derived microglial cells. Neurochem Int 1996;29:55-64

Wu DC, Teismann $\mathrm{P}$, Tieu $\mathrm{K}$, Vila $\mathrm{M}$, Jackson-Lewis $\mathrm{V}$ Ischiropoulos $\mathrm{H}$, Przedborski S. NADPH oxidase mediates oxidative stress in the 1-methyl-4-phenyl-1,2,3,6-tetrahydropyridine model of Parkinson's disease. Proc Natl Acad Sci USA 2003;100:6145-50

Wu DC, Re DB, Nagai M, Ischiropoulos H, Przedborski S. The inflammatory NADPH oxidase enzyme modulates motor neuron degeneration in amyotrophic lateral sclerosis mice. Proc Natl Acad Sci USA 2006;103:12132-7

Zipp F, Aktas O. The brain as a target of inflammation: common pathways link inflammatory and neurodegenerative diseases. Trends Neurosci 2006;29:518-27 